\title{
Alkylation of toluene with isopropyl alcohol over SAPO-5 catalyst
}

\author{
SREEDEVI UPADHYAYULA \\ Department of Chemical Engineering, Indian Institute of Technology Delhi, Hauz Khas, \\ New Delhi 110016 \\ e-mail: sreedevi@chemical.iitd.ac.in
}

MS received 25 August 2008; revised 11 December 2008

\begin{abstract}
Isopropylation of toluene with isopropyl alcohol was studied over the large pore silicon alumino phosphate molecular sieves (SAPO-5) with varying Si content. Toluene conversion was found to increase with increase in the $\mathrm{Si}$ of the catalysts. The effect of temperature on yields of cymene was studied in the range of 453 to $553 \mathrm{~K}$. The highest yield and selectivity for cymene was observed at $513 \mathrm{~K}$. Good cymene selectivity was confirmed with the absence of $n$-propyl toluene in the product. The catalyst activity, in terms of $p$-cymene selectivity, remained almost stable during a nine hour time on stream run. Enhanced cymene selectivity and decreased $p$-cymene selectivity was observed with increase in the toluene to isopropyl alcohol feed mole ratio from 2 to 8 . Power law model and $\mathrm{L}-\mathrm{H}-\mathrm{H}-\mathrm{W}$ model fitted the experimental data well and are used to explain the kinetics of this reaction.
\end{abstract}

Keywords. Cymene; $p$-cymene; isopropylation; toluene; SAPO-5.

\section{Introduction}

In most of the industrial alkylation processes, catalysts such as mineral acids and anhydrous $\mathrm{AlCl}_{3}$ are used. The relatively high volumes of catalyst in these processes make them highly polluting. Also, a lot of energy is consumed, annually, to separate the acid catalyst from product. In recent times, considerable attention is focused on the selective production of para-dialkylbenzenes, like xylene, ethyl toluene, diethyl benzene via alkylation reactions catalysed by shape selective zeolites or other solid acid catalysts. ${ }^{1-5}$ Isopropyl toluene (cymene), in particular, the para isomer of it, is one such commercially important dialkylbenzene. It is used as an important starting material for the production of a range of intermediates and end products, such as cresols, isopropyl phenols, pesticides, fungicides, fragrances, pharmaceuticals, herbicides, heat transfer media, etc.

The alkylation of toluene over zeolites follows the well known Friedel-Crafts mechanism. It proceeds by direct ortho-para attack of the ring followed by positional isomerisation producing the meta isomer. ${ }^{6,7}$ Modification of a zeolite can alter the chemical properties of the catalytic centers so that it ceases to partially or completely facilitate isomerisation, thus, increasing the selective yield of the important para-isomer. Fraenkel and Levy $^{8}$ studied toluene isopropylation reaction on medium pore HZSM-5 zeolites differing in crystal size and morphology and proposed a reaction mechanism. They also compared the product distribution of the reaction over these medium pore zeolites with that over the large pore $\mathrm{HY}$ and HM. While HM and HY yielded cymenes selectively, a very high proportion of $n$-propyl toluene was observed over HZSM-5. Parikh et al $l^{9}$ studied the same reaction over zeolites varying in pore size distribution, crystal size and proposed a more realistic mechanism on ZSM-5. Guo et $a l^{10}$ investigated the alkylation of toluene by propylene over HZSM12 zeolite and obtained cymene isomers with a near thermodynamic equilibrium composition. Čejka et $a l^{11}$ investigated the factors controlling para-selectivity in the alkylation of toluene with isopropyl alcohol over molecular sieves possessing different acidity (Al- and Fe-silicates) and structure type (Y, mordenite and MFI structure). They concluded that the MFI structure and high acidity of the molecular sieves tend to increase the $n$-propyl toluene yield. According to them, the alkylation activity does not follow the acidity, but the reaction is controlled by the desorption/transport rate of bulky cymenes and cumene. Toluene isopropylation on beta zeolites has been reported by Reddy et al. ${ }^{12}$ These zeolites were observed to be highly stable but possess negligible shape selectivity in toluene isopropylation. Witchter- 
Table 1. Synthesis gel ratios and crystallization conditions for SAPO-5 catalysts.

\begin{tabular}{lcccccccc}
\hline & \multicolumn{5}{c}{ Gel composition } & & \multicolumn{2}{c}{ Reaction conditions } \\
\cline { 2 - 5 } Sample & $\mathrm{Al}_{2} \mathrm{O}_{3}$ & $\mathrm{P}_{2} \mathrm{O}_{5}$ & $\mathrm{SiO}_{2}$ & $\mathrm{R}$ & $\mathrm{H}_{2} \mathrm{O}$ & & Temp $\left({ }^{\circ} \mathrm{C}\right)$ & Time (h) \\
\hline SAPO-5b & 1.0 & 0.975 & 0.05 & $1 \cdot 1$ & 55 & & 200 & 24 \\
SAPO-5c & $1 \cdot 0$ & 0.95 & $0 \cdot 10$ & $1 \cdot 1$ & 55 & & 200 & 24 \\
SAPO-5d & $1 \cdot 0$ & 0.925 & $0 \cdot 20$ & $1 \cdot 1$ & 55 & & 200 & 24 \\
\hline
\end{tabular}

$\mathrm{R}$, Template; di- $n$-propylamine for SAPO-11 and tri-ethylamine for SAPO-5S

lova et $a l^{13}$ studied the selective formation of $p$ cymene over $\mathrm{Al}$ and Fe silicates.

From the above discussion, it is evident that although toluene isopropylation has been studied over a variety of catalysts, mostly zeolites, yet, no attempt has been made till date to use silicon substituted alumino phosphates (SAPOs) as catalysts for this reaction. Hence, the present study was undertaken with an objective to investigate the effect of various process parameters and the kinetics of toluene alkylation with isopropyl alcohol over SAPO-5 catalyst.

\section{Experimental}

\subsection{Materials}

The SAPO-5 molecular sieves SAPO-5b, SAPO-5c and SAPO-5d respectively were prepared following procedure described by Lok et $a l^{14}$ and characterized as detailed below.

Three SAPO-5 samples with $0 \cdot 05,0 \cdot 1$ and $0.2 \mathrm{M}$ silica in the starting gel were synthesized and these were designated as SAPO-5b, SAPO-5c and SAPO$5 \mathrm{~d}$ respectively. The gel ratios and synthesis conditions are given in table 1. In a typical synthesis (for SAPO-5c), 34.68 g of Psuedoboehmite was added to dilute phosphoric acid $\left(54.765 \mathrm{~g}\right.$ of $\mathrm{H}_{3} \mathrm{PO}_{4}$ in $160 \mathrm{~g}$ of water) slowly, in a constantly stirred autoclave. After the addition of the aluminium source, stirring was continued at least for one hour. Subsequently, $\mathrm{SiO}_{2}$ in $47.6 \mathrm{~g}$ of water was added to the above and stirring continued for another hour. To this mixture, $26.562 \mathrm{~g}$ of tri-ethylamine was added under vigorous stirring. The gel thus obtained was stirred further, filled in a teflon lined autoclave and kept for heating at $200^{\circ} \mathrm{C}$ under autogeneous pressure. After heating for $24 \mathrm{~h}$, the crystalline product was separated from mother liquor and repeatedly washed with distilled water. The crystals were dried for $12 \mathrm{~h}$ at $110^{\circ} \mathrm{C}$ and subsequently, calcined at $550^{\circ} \mathrm{C}$ for $12 \mathrm{~h}$ to remove the template.
The as synthesized SAPO-5 molecular sieves were characterized for their phase purity, crystallinity and morphology using X-ray diffraction (XRD) and scanning electron microscopy (SEM). They were found to be highly crystalline pure phases.

Toluene and isopropyl alcohol (IPA) used in the present study were obtained from E Merck (India) Ltd. and Qualigens Fine Chemicals, India respectively and were of 'Analytical Reagent' grade.

\subsection{Procedure}

The experiments were conducted in a down flow fixed bed tubular reactor $(15 \mathrm{~mm}$ i.d.) with $2 \mathrm{~g}$ of catalyst loaded in the reactor. Nitrogen was used as the carrier gas. Toluene to isopropyl alcohol mole ratio in the feed was maintained at $6: 1$ during the experiments. A feed flow rate of $6.8 \times 10^{-3} \mathrm{~kg} / \mathrm{h}$ and more was used for the kinetic study in order to eliminate external mass transfer effects. The products of the reaction were analysed with a SHIMADZU 15A gas chromatograph fitted with a 'CP-Xylenes' capillary $(50 \mathrm{~m}, 0.32 \mathrm{~mm}$ i.d.) column using a flame ionization detector (FID).

\section{Results and discussions}

In the present study, toluene was used in excess and isopropyl alcohol (IPA) was the limiting reactant. Hence, pseudo first order kinetics were investigated based on isopropyl alcohol.

The term 'conversion of IPA' used in the kinetic studies is defined as

IPA conversion $(\mathrm{mol} \%)=$ (moles of IPA consumed/ moles of IPA fed per unit time) $\times 100$.

The terms toluene conversion, cymene selectivity, $p$ cymene selectivity are defined as follows: 
Table 2. Comparison of activities of various SAPO-5 catalysts in toluene isopropylation reaction ${ }^{\mathrm{a}}$.

\begin{tabular}{|c|c|c|c|c|c|}
\hline Catalyst & SAPO-5b & SAPO-5c & SAPO-5d & SAPO-11 & SAPO- $11^{\ddagger}$ \\
\hline \multicolumn{6}{|l|}{ Product yield (wt\%) } \\
\hline Aliphatics & $b d^{*}$ & $b d^{*}$ & $b d^{*}$ & $2 \cdot 01$ & 1.67 \\
\hline Benzene & $b d^{*}$ & $b d^{*}$ & 0.056 & $0 \cdot 12$ & $0 \cdot 10$ \\
\hline Toluene & $81 \cdot 24$ & $80 \cdot 13$ & $78 \cdot 57$ & $97 \cdot 30$ & $94 \cdot 17$ \\
\hline $\mathrm{C}_{8}$ aromatics & $b d^{*}$ & $b d^{*}$ & $b d^{*}$ & $b d^{*}$ & $b d^{*}$ \\
\hline Cumene & $b d^{*}$ & $b d^{*}$ & 0.074 & $b d^{*}$ & $b d^{*}$ \\
\hline$p$-Cymene & $6 \cdot 99$ & $7 \cdot 34$ & 7.91 & $0 \cdot 565$ & $3 \cdot 52$ \\
\hline$m$-Cymene & $8 \cdot 85$ & $10 \cdot 52$ & $11 \cdot 40$ & $b d^{*}$ & 0.43 \\
\hline$o$-Cymene & 1.99 & 1.51 & 1.44 & $b d^{*}$ & 0.08 \\
\hline DIPT & 0.83 & 0.70 & 0.56 & $b d^{*}$ & 0.03 \\
\hline Cymenes (wt\%) & $17 \cdot 92$ & $19 \cdot 37$ & $20 \cdot 75$ & 0.57 & 4.03 \\
\hline Cymenes sel (wt\%) & $95 \cdot 57$ & $96 \cdot 51$ & $96 \cdot 80$ & $82 \cdot 12$ & $96 \cdot 88$ \\
\hline$p$-Cymene sel (wt\%) & $37 \cdot 25$ & 37.89 & $38 \cdot 14$ & 99.99 & $87 \cdot 35$ \\
\hline
\end{tabular}

${ }^{a}$ Conditions: temperature, $473 \mathrm{~K}$; WHSV, $3 \mathrm{~h}^{-1}$; toluene/IPA mole ratio, 6:1 DIPT: diiospropyl toluene; *below detectable limits; \#: at $573 \mathrm{~K}$

Toluene conversion $(\mathrm{wt} \%)=$

[(toluene in product)/toluene in feed $] \times 100$

Cymene selectivity $(\mathrm{wt} \%)=$

[(cymenes in product)/total aromatics

(excluding toluene) in product $] \times 100$

$p$-cymene selectivity $(\mathrm{wt} \%)=[($ yield of $p$-cymene $) /$ total yield of cymenes] $\times 100$.

\subsection{Effect of acidity}

It is known that acidity of SAPO-5 catalysts depends upon the amount and mode of silicon substituted into the framework and increases with increase in $\mathrm{Si}$ content of the catalyst. ${ }^{15}$ Studies based on ${ }^{29} \mathrm{Si}$ NMR and acidity measurements show that the acidity of the catalysts is in order of SAPO-5d $>$ SAPO-5c $>$ SAPO-5b. The cymene yield also decreased in the same order for the SAPO-5 series. Table 2 shows the effect of variation of Si content on cymene yield, selectivity. The yields of meta- and para-cymenes increased with the increase in Si content while that of the ortho-isomer decreased. Also, the cymene selectivity increased from $95.57 \%$ over SAPO-5b to $96.8 \%$ over SAPO- $5 \mathrm{~d}$. In particular, p-cymene selectivity, increased from $37.89 \%$ to $40.73 \%$ with the increase in $\mathrm{Si}$ content which can be attributed to the increased conversion of toluene over SAPO-5b compared to SAPO-5d.

The yields of diisopropyl toluenes (DIPTs) were also observed to decrease with the increase in the $\mathrm{Si}$ content. This could be because of the absence of sufficient number of active acid sites for realkylation of cymenes. It is also observed from the product distribution pattern in table 2 that small quantities of cumene and benzene are formed due to toluene disproportionation and benzene isopropylation side reactions. These side reactions do not seem to take place over the catalysts with lower $\mathrm{Si}$ content or acidity. Despite these side reactions, the cymene selectivity is the highest over SAPO-5d among the SAPO-5 catalysts tested. Hence, this catalyst was chosen for detailed kinetic investigations.

The same table also compares the activity of the medium pore SAPO-11 catalyst with SAPO-5. It has been observed that the SAPO-11 which has milder acidity than SAPO-5 is not active in the isopropylation of toluene even at a higher reaction temperature $(573 \mathrm{~K})$ yielding only $4.03 \mathrm{wt} \%$ cymenes.

\subsection{Effect of temperature on cymene yield and selectivity}

Figure 1 summarizes the effect of temperature on the yield and selectivity of cymenes. The yield and total cymene selectivity has been observed to rise with increasing temperature up to an optimum temperature of $513 \mathrm{~K}$. Thereafter, a decrease in the cymene yield and selectivity was observed. At lower temperatures, the $p$-cymene selectivity is $40 \%$, above that of the equilibrium value, but with the increase in temperature, the $p$-cymene yield reduces and moves towards the equilibrium value of $28 \%$ (within 
the temperature range considered). The decrease in $p$-cymene selectivity with temperature is attributed to the isomerization reaction to form $m$-cymene which is favoured at higher temperature.

However, the $o$-cymene was found to be always much lower than the equilibrium value. This can be attributed to the isomerization to $m$-cymene, favoured at higher temperatures, and also, the diffusional resistance in the pores of the catalyst. The selectivity to $m$-cymene reaches a maximum of $12.5 \%$ at $513 \mathrm{~K}$ and falls with further increase in temperature. At temperatures higher than $513 \mathrm{~K}$, the active sites for the isomerisation of $p$-cymene to $m$-cymene could be blocked by coke formed by side reactions like alkyl aromatic cracking.

\subsection{Effect of time on stream on toluene conversion} and cymene selectivity

Figure 2 shows the variation of activity of the catalyst during a $9 \mathrm{~h}$ time on stream run. The toluene conversion was observed to decrease by about $5 \%$ at the end of $8 \mathrm{~h} 30 \mathrm{~min}$ time on stream. The cymene selectivity almost remains constant until the end of $6 \mathrm{~h}$ and then drops by 3-5\%. Selectivity to $p$ cymene is observed to remain almost constant at $40 \%$ throughout the run. The decrease in the conversion of toluene is attributed to the deactivation of the catalysts due to coke formation reaction and subsequent pore blocking. The deactivation kinetics follow a parallel mechanism in the aromatics alkylation

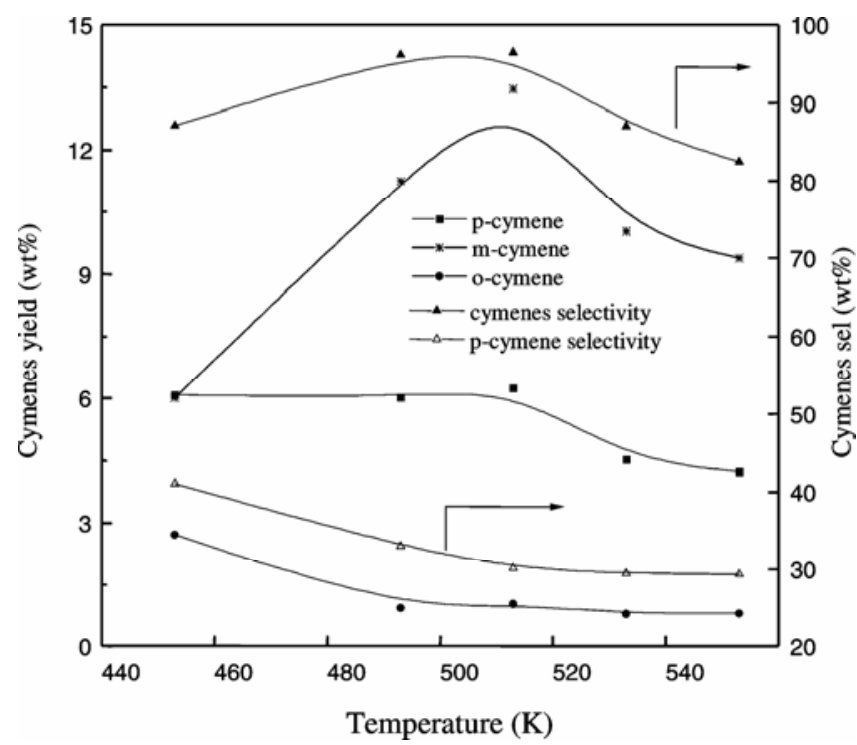

Figure 1. Effect of temperature on cymenes yield and selectivity. with isopropyl alcohol over SAPO-5 and reported in an earlier paper. ${ }^{5}$ The experimental data for the kinetic study was collected within the time of stable activity of the catalyst.

\subsection{Effect of feed mole ratio on toluene conversion and cymene selectivity}

Figure 3 shows the effect of feed mole ratio on toluene conversion, total cymene selectivity and $p$-cymene

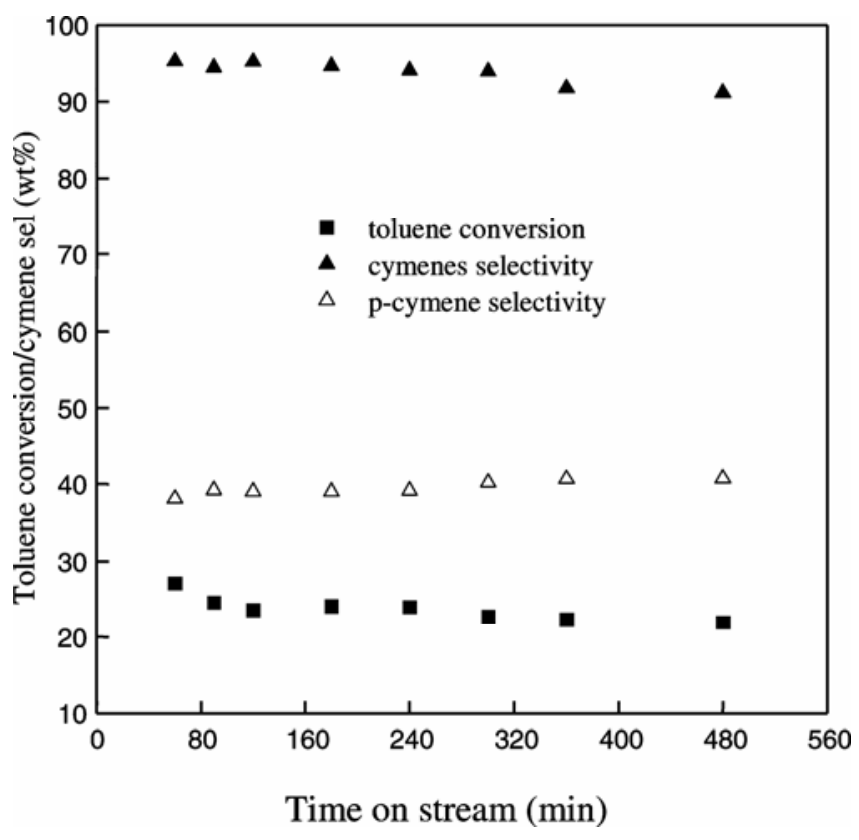

Figure 2. Effect of time on stream on toluene conversion and cymene selectivity.

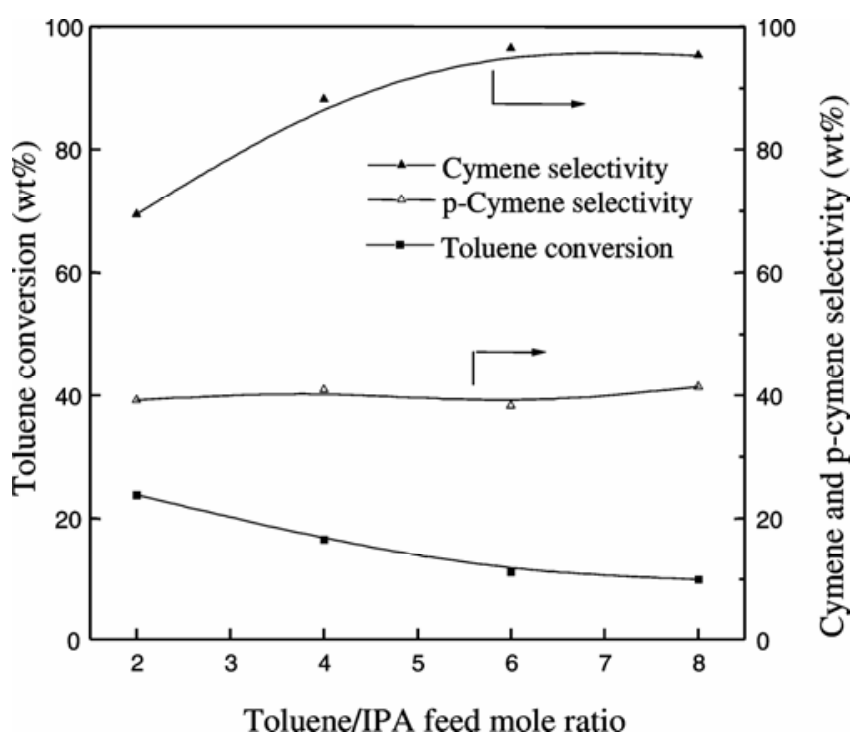

Figure 3. Effect of feed composition on toluene conversion and cymene selectivity. 
Table 3. Effect of WHSV on cymenes yield and selectivity over SAPO-5 $5^{\text {a }}$

\begin{tabular}{|c|c|c|c|c|}
\hline WHSV $\left(h^{-1}\right)$ & $3 \cdot 0$ & $6 \cdot 3$ & $9 \cdot 6$ & $12 \cdot 0$ \\
\hline \multicolumn{5}{|c|}{ Product distribution (wt\%) } \\
\hline Aliphatics & $b d^{*}$ & $b d^{*}$ & $b d^{*}$ & $0 \cdot 11$ \\
\hline Toluene & $80 \cdot 13$ & $82 \cdot 02$ & $81 \cdot 59$ & $82 \cdot 64$ \\
\hline$p$-cymene & $7 \cdot 34$ & $6 \cdot 62$ & $7 \cdot 15$ & $6 \cdot 45$ \\
\hline$m$-cymene & $10 \cdot 52$ & $8 \cdot 55$ & $8 \cdot 27$ & $6 \cdot 37$ \\
\hline$o$-cymene & $1 \cdot 51$ & $2 \cdot 08$ & $2 \cdot 13$ & $3 \cdot 03$ \\
\hline DIPTs & $0 \cdot 70$ & 0.73 & $0 \cdot 86$ & $1 \cdot 40$ \\
\hline Cymenes (wt\%) & $19 \cdot 37$ & $17 \cdot 26$ & $17 \cdot 55$ & $15 \cdot 85$ \\
\hline Cymenes sel (wt\%) & $96 \cdot 51$ & $95 \cdot 95$ & $95 \cdot 32$ & 91.88 \\
\hline$p$-Cymene sel (wt\%) & $37 \cdot 89$ & $38 \cdot 84$ & 40.73 & 40.73 \\
\hline
\end{tabular}

selectivity. Toluene conversion decreases with lower percentage of isopropyl alcohol in the feed which is as expected. At lower toluene to isopropyl alcohol ratios in the feed, the cymenes selectivity decreased from $95 \%$ (at 6-8 mole ratio of toluene to IPA) to $70 \%$ (at 2 mole ratio of toluene and IPA) due to the formation of undesirable side products by oligomerisation reactions of propylene formed by dehydration of isopropyl alcohol whose concentration is increased in the feed. At higher toluene to isopropyl alcohol mole ratios, these undesirable side products are reduced, showing an increase in the total cymene selectivity. The $p$-cymene selectivity, however, remains almost constant with the increase in toluene to isopropyl alcohol mole ratio.

\subsection{Effect of weight hourly space velocity (WHSV)}

Table 3 summarizes the effect of space velocity on product distribution. The cymenes yield and selectivity were observed to decrease with increased space velocity due to decrease in residence time in the reactor. However, the $p$-cymene selectivity was observed to increase at higher space velocities probably, due to the suppression of the $p$-cymene isomerisation to the meta isomer. From the product distribution, we see that at the highest space velocity, even aliphatics are present in the product due to incomplete conversion of isopropyl alcohol.

\subsection{Kinetic study}

To determine the reaction rate parameters for the conversion of isopropyl alcohol, a differential plug flow reactor was assumed, since, the conversion of isopropyl alcohol in the temperature range (401$415 \mathrm{~K})$ studied, were below $30 \%$. At this lower temperature range, diisopropyl toluenes or other side products were not detected in the product stream. The reaction scheme in this low temperature range can be written as

Main reaction: toluene + IPA $\rightarrow p$-cymene + water

Secondary reactions:

$$
\begin{aligned}
& p \text {-cymene } \rightarrow m \text {-cymene (isomerisation) } \\
& \text { IPA } \rightarrow \text { propylene }+ \text { water } \\
& \text { Propylene } \rightarrow \text { aliphatics (oligomerisation) } \\
& \text { IPA }+ \text { toluene } \rightarrow \text { coke }
\end{aligned}
$$

3.6a Homogeneous kinetic model: Using the first order rate equation for a differential plug flow reactor, the rate of disappearance of isopropyl alcohol may be written as

$$
-r_{A}=\mathrm{d} X_{A} / \mathrm{d} \tau=\mathrm{d} X_{A} / \mathrm{d}\left(W / F_{\mathrm{AO}}\right)=k C_{\mathrm{A}},
$$

where $W$ is the weight of the catalyst in kilograms, $F_{\mathrm{AO}}$ is the feed isopropyl alcohol flow rate in $\mathrm{kgmol} / \mathrm{h}, X_{\mathrm{A}}$ is the fractional conversion of IPA, $\tau$ is the space time in $\mathrm{kg} \mathrm{h} / \mathrm{kg} \mathrm{mol}, k$ is the rate constant and $C_{\mathrm{A}}$ is the concentration of isopropanol in $\mathrm{kgmol} / \mathrm{m}^{3}$. Figure 4 shows the isopropyl alcohol conversion at different space times at $401 \mathrm{~K}, 405 \mathrm{~K}$, and $408 \mathrm{~K}$. The straight lines confirm a differential conversion at all these three temperatures.

Integration of equation (1) yields,

$$
-\ln \left(1-X_{\mathrm{A}}\right)=k C_{\mathrm{Ao}}\left(W / F_{\mathrm{AO}}\right) \text {. }
$$

The plot of $-\ln \left(1-X_{\mathrm{A}}\right)$ against $W / F_{\mathrm{AO}}$ over SAPO-5d gave a straight line passing through the origin as illustrated in figure 5 at various temperatures. 
The rate of isopropyl alcohol conversion is, therefore, first order with respect to isopropyl alcohol, which is the limiting reactant, and is of zero order with respect to toluene which is in large excess. From the slopes of the lines and with $C_{\mathrm{Ao}}$ as $1.39 \times$ $10^{-3} \mathrm{~kg} \mathrm{~mol} / \mathrm{m}^{3}$, the rate constant $(k)$ was calculated to be $3.50 \mathrm{~m}^{3} / \mathrm{kg} \mathrm{h}$ at $415 \mathrm{~K}$, with $C_{\mathrm{Ao}}$ as $1.42 \times$ $10^{-3} \mathrm{~kg} \mathrm{~mol} / \mathrm{m}^{3}$ rate constant $(k)$ was $2.74 \mathrm{~m}^{3} / \mathrm{kg}$ h at $408 \mathrm{~K}$ and with $C_{\mathrm{Ao}}$ as $1.45 \times 10^{-3} \mathrm{~kg} \mathrm{~mol} / \mathrm{m}^{3}$ rate constant $(k)$ was $2.07 \mathrm{~m}^{3} / \mathrm{kg} \mathrm{h}$ at $401 \mathrm{~K}$ on SAPO-5d.

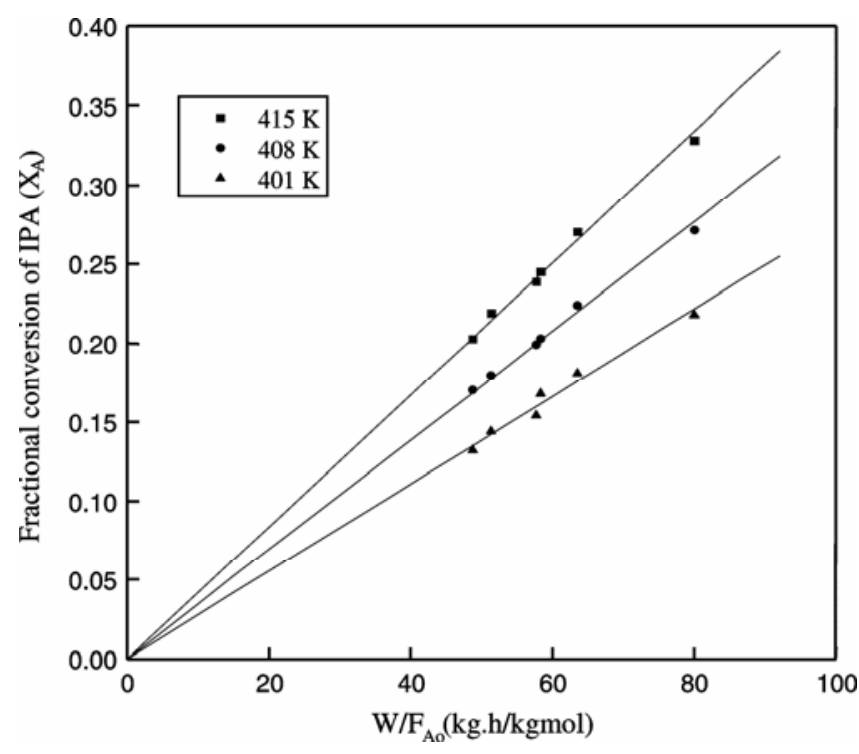

Figure 4. Effect of space time on IPA conversion.

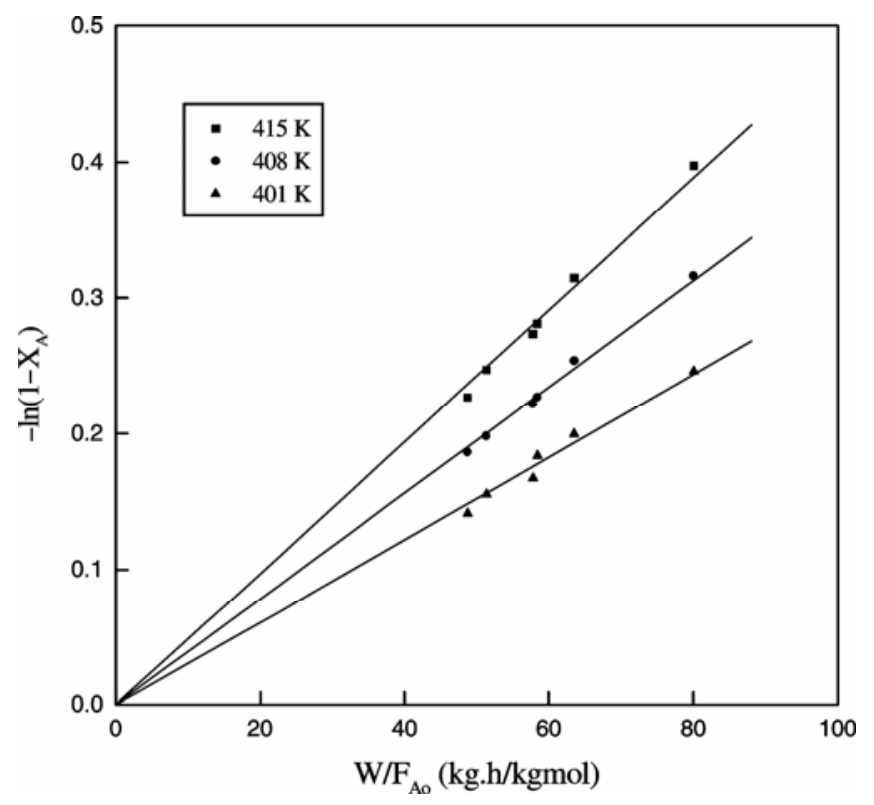

Figure 5. First order kinetic plot for toluene isopropylation over SAPO-5d.
Employing the power law equation from Power law kinetics, ${ }^{16}$ the rate of the reaction for the present system would be,

$$
-r_{A}=A \exp \left(-E_{\mathrm{a}} / \mathrm{RT}\right) C_{\mathrm{A}},
$$

where $E_{\mathrm{a}}$ is the apparent activation energy and $A$ is the Arrhenius frequency factor. Since, for a reaction in a differential plug flow reactor, $-r_{\mathrm{A}}=\left(F_{\mathrm{AO}} / W\right) X_{\mathrm{A}}$, (3) can be modified as

$$
\left(F_{\mathrm{AO}} / W\right) X_{\mathrm{A}}=A \exp \left(-E_{\mathrm{a}} / R T\right) C_{\mathrm{A}},
$$

writing $C_{\mathrm{A}}$ in terms of initial concentration and fractional conversion of isopropyl alcohol and rearranging the equation, we obtain

$$
\ln \left(X_{\mathrm{A}} / 1-X_{\mathrm{A}}\right)=\ln A_{\mathrm{o}}-E_{\mathrm{a}} / R T,
$$

where $A_{\mathrm{o}}$ is the apparent Arrhenius frequency factor which includes a constant such as $W / F_{\mathrm{AO}}$ as well as $C_{\mathrm{Ao}}$ (initial concentration of isopropyl alcohol) and $E_{\mathrm{a}}$ is the apparent activation energy. From (5), it is clear that a plot of $\ln \left(X_{\mathrm{A}} / 1-X_{\mathrm{A}}\right)$ against $1 / T$ would give a straight line from which $E_{\mathrm{a}}$ and the $A_{\mathrm{o}}$ can be obtained.

The Arrhenius plot for the isopropyl alcohol conversion employing (5) is shown in figure 6 . The value of $E_{\mathrm{a}}$ from the plot is $55.36 \mathrm{~kJ} / \mathrm{mol}$ and $A_{\mathrm{o}}$ is $4.538 \times 10^{6}$. The apparent activation energy evaluated is comparable to the activation energies reported by earlier workers for alkylation of toluene over zeolites. Parikh et $a l^{17}$ reported an apparent activation energy of $50 \mathrm{~kJ} / \mathrm{mol}$ for toluene isopropylation

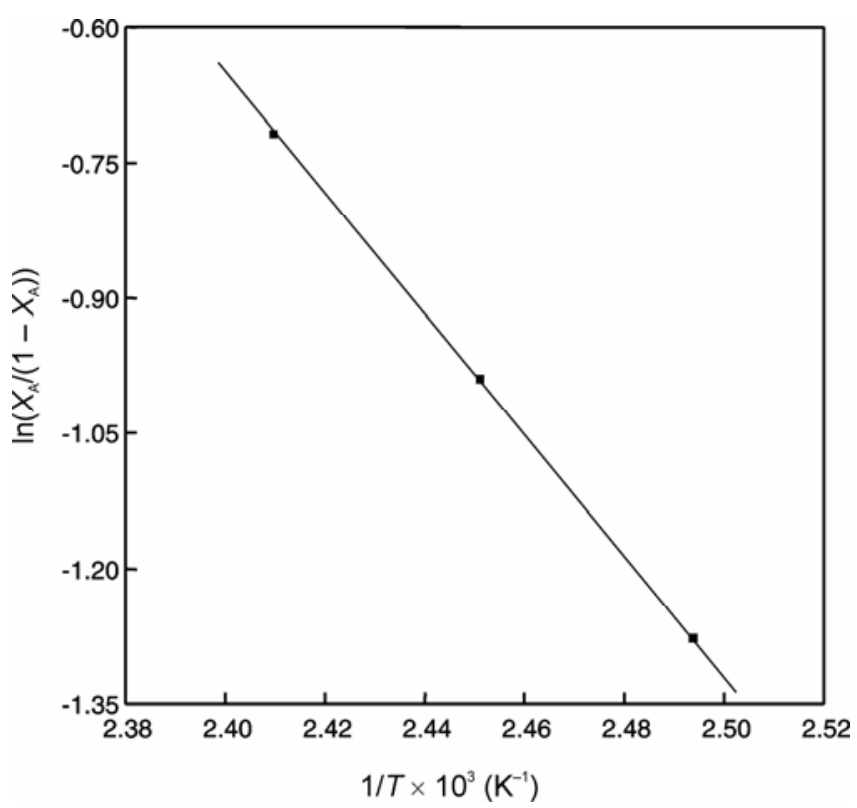

Figure 6. Arrhenius plot with SAPO-5d as the catalyst. 
Table 4. Parameter estimation for heterogeneous kinetic model.

\begin{tabular}{lcccccc}
\hline Temp. (K) & $\begin{array}{c}k \text { (rate constant, } \\
\mathrm{kg} \mathrm{mol} / \mathrm{kg} \mathrm{h})\end{array}$ & $K_{\mathrm{A}}\left(\mathrm{atm}^{-1}\right)$ & $K_{\mathrm{B}}\left(\mathrm{atm}^{-1}\right)$ & $K_{\mathrm{C}}\left(\mathrm{atm}^{-1}\right)$ & $K_{\mathrm{D}}\left(\mathrm{atm}^{-1}\right)$ & $\left.\begin{array}{c}K_{\mathrm{r}}(\mathrm{kg} \mathrm{mol} / \\
\mathrm{kg} \mathrm{h} \mathrm{atm}\end{array}\right)$ \\
\hline 401 & 0.734 & 35.8486 & 0.0378 & 30.6653 & 0.0776 & 1.00515 \\
408 & 0.805 & 33.7507 & 0.0356 & 19.7297 & 0.021 & 1.00149 \\
415 & 0.8567 & 26.2048 & 0.0345 & 0.0142 & 0.019 & 1.00152 \\
\hline
\end{tabular}

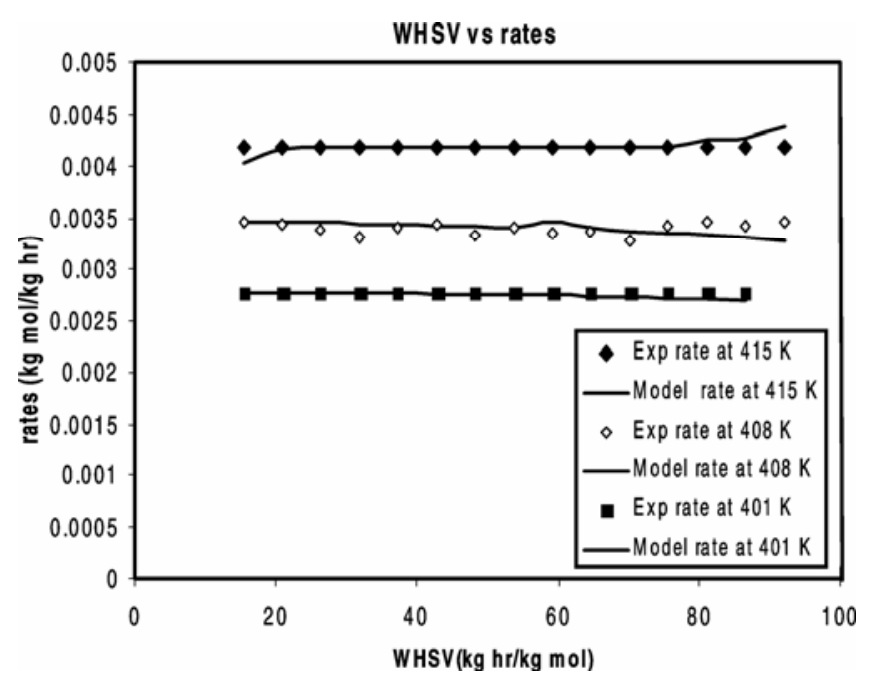

Figure 7. Comparison of experimental and heterogeneous model predicted rates.

over ZSM-5 and a rate constant of $1.714 \mathrm{~g} \mathrm{~mol} /$ $\mathrm{h} /$ gcat $/ \mathrm{atm}^{2}$ at $498 \mathrm{~K}$ which is only very slightly higher than our rates due to the higher temperature range considered by them. Bhat et $a l^{18}$ reported an apparent activation energy of $63 \mathrm{~kJ} / \mathrm{mol}$ for toluene ethylation over HZSM-8 with only a slightly higher rate constant of $1.862 \mathrm{~mol} / \mathrm{g}$ atm $\mathrm{h}$ at much higher temperature of $673 \mathrm{~K}$.

3.6b Heterogeneous kinetic model: The heterogeneous model is based on the Langmuir-Hinshelwood-Hougen-Watson kinetics wherein the main reaction forming $p$-cymenes and water is catalysed by the active sites on the catalyst. According to this, toluene and isopropyl alcohol are chemisorbed on dual adjacent active sites of the catalyst to form different carbonium ions. Both chemisorbed IPA and toluene react with each other to form cymenes and water followed by desorption of cymenes and water from the active sites. Here, the dual-site surface reaction is the rate controlling step. These steps are summarized as given below.

$$
A \delta+B \delta \underset{K_{r-1}}{\stackrel{K_{r}}{\rightleftarrows}} C \delta+D \delta
$$

$$
C \delta \stackrel{K_{\mathrm{c}}}{\underset{K_{-\mathrm{c}}}{\rightleftarrows}} C+\delta \quad D \delta \stackrel{K_{\mathrm{d}}}{\underset{K_{-\mathrm{d}}}{\rightleftarrows}} D+\delta
$$

$A=\mathrm{IPA} ; B=$ toluene; $C=p$-cymene; $D=$ water and $\delta=$ vacant active site.

The overall rate expression following the surface reaction controlling dual site mechanism is expressed in concentration terms $\left(C_{\mathrm{A}}, C_{\mathrm{B}}, C_{\mathrm{C}}, C_{\mathrm{D}}\right)$ as

$$
-r_{s}=\frac{k\left(K_{A} \cdot K_{B} \cdot C_{B} \cdot C_{A}-K_{D} \cdot K_{C} \cdot C_{C} \cdot C_{D} / K_{r}\right)}{\left(1+K_{A} \cdot C_{A}+K_{B} \cdot C_{B}+K_{D} \cdot C_{D}+K_{C} \cdot C_{C}\right)^{\wedge} 2}
$$

where $K_{\mathrm{A}}=$ equilibrium adsorption constant of IPA; $K_{\mathrm{B}}=$ equilibrium adsorption constant of toluene; $K_{\mathrm{C}}=$ equilibrium desorption constant of $p$-cymene and $K_{\mathrm{D}}=$ equilibrium desorption constant of water, $K_{r}=$ equilibrium constant for overall dual-site surface reaction.

The parameters are estimated by non-linear regression technique and summarized for the three temperatures in table 4 . The model predicted rates agree well with the experimental rates as shown in figure 7.

\subsection{Thermodynamics and equilibrium constant evaluation}

The equilibrium constant for a chemical reaction is defined as

$$
\Delta F^{\circ}=-R T \ln K_{\mathrm{p}},
$$

where $\Delta F^{\circ}$ is the free energy change in the reaction, $R$ is the universal gas constant, $K_{\mathrm{p}}$ is the thermodynamic equilibrium constant based on pressure.

Now, by Van Krevlen method, the free energy of formation of a compound at any temperature $T$ is given by

$$
\Delta F_{\mathrm{f}}^{\circ}=A+B T,
$$

where $A$ and $B$ are constants as given in the standard tables by Reid and Sherwood. ${ }^{19}$

The equilibrium constants for the reversible isomerisation reaction of $p$-cymene to $m$-cymene at $401 \mathrm{~K}$, $408 \mathrm{~K}$ and $415 \mathrm{~K}$ were determined as follows: 


$$
\begin{aligned}
& \text { At } 401 \mathrm{~K} \\
& \Delta F_{\mathrm{f}}^{\circ}(\text { for } p \text {-cymene })=\mathrm{A}+\mathrm{BT}=0.93 \mathrm{kcal} / \mathrm{gmol} \\
& \Delta F_{\mathrm{f}}^{\circ}(\text { for } m \text {-cymene })=-0.31 \mathrm{kcal} / \mathrm{gmol} \text {. }
\end{aligned}
$$

Therefore, (6) can be written as

$$
\Delta F^{\circ}=\Delta F_{\mathrm{f}}^{\circ}(\text { products })-\Delta F_{\mathrm{f}}^{\circ}(\text { reactants })=-R T \ln K_{\mathrm{p}} .
$$

Substituting the above values of $\Delta F_{\mathrm{f}}{ }^{\circ}$ of $p$-cymene, $m$-cymene and $R=1.987 \mathrm{cal} / \mathrm{mol} \mathrm{K}$.

$$
K_{\mathrm{p}}=1.00516 \mathrm{~atm}^{-1} \text { at } 401 \mathrm{~K} \text {. }
$$

Similarly, the values of $K_{\mathrm{p}}$ are $1.00153 \mathrm{~atm}^{-1}$ at $408 \mathrm{~K}$ and $1.0015 \mathrm{~atm}^{-1}$ at $415 \mathrm{~K}$.

\section{Conclusions}

Alkylation of toluene with isopropyl alcohol was studied over a variety of SAPO-5 molecular sieves. Toluene conversion was found to increase with increase in Si content of the SAPO-5 catalysts. The yield of cymenes went through a maximum as the reaction temperature was increased from $453 \mathrm{~K}$ to $553 \mathrm{~K}$. The highest yield and selectivity for cymene was observed at $513 \mathrm{~K}$. Undesirable side product like $n$-propyl toluene was absent over this catalyst. The $p$-cymene selectivity remained almost constant during a nine hour time on stream run. A change in toluene to isopropyl alcohol feed ratio from 2 to 8 resulted in enhanced cymene selectivity and decreased $p$-cymene selectivity. Both Power law model, assuming pseudo-first order reaction, and L$\mathrm{H}-\mathrm{H}-\mathrm{W}$ model fit the experimental data fairly well

\begin{tabular}{|c|c|}
\hline $\mathrm{Al}$ & Alumina \\
\hline A & Arrehenius frequency factor \\
\hline$A_{\mathrm{o}}$ & Apparent Arrehenius frequency factor \\
\hline$C_{\mathrm{A} 0}$ & $\begin{array}{l}\text { Concentration of isopropanol in feed, } \\
\mathrm{kgmol} / \mathrm{m}^{3}\end{array}$ \\
\hline$C_{\mathrm{A}}$ & Concentration of isopropanol, $\mathrm{kgmol} / \mathrm{m}^{3}$ \\
\hline DIPT & 2,4-diisopropyl toluene \\
\hline$E_{\mathrm{a}}$ & Apparent activation energy, $\mathrm{kJ} / \mathrm{mol}$ \\
\hline$F_{\text {Ao }}$ & Feed flow rate of isopropanol, $\mathrm{kg} \mathrm{mol} / \mathrm{h}$ \\
\hline$F_{\mathrm{f}}$ & Free energy of formation in $\mathrm{Kcal} / \mathrm{g} \mathrm{mol}$ \\
\hline HM & Hydrogen Mordenite \\
\hline IPA & Isopropanol \\
\hline
\end{tabular}
in the temperature range of $401-415 \mathrm{~K}$. The activation energy compares well with earlier reports on similar reactions over molecular sieves.

\section{Nomenclature}

$k \quad$ Pseudo-first order rate constant in $\mathrm{m}^{3} / \mathrm{kg}$.

- Thermodynamic equilibrium constant based on pressure $\mathrm{atm}^{-1}$

$m \quad$ Meta

$o \quad$ Ortho

$p \quad$ Para

$R \quad$ Ideal gas law constant, $82.06 \mathrm{~cm}^{3}$ atm/gm-mol. $\left({ }^{\circ} \mathrm{K}\right)$

$r_{\mathrm{A}} \quad$ Rates of reaction of isopropanol, kgmol/ $\mathrm{kg}$ of cat. $\mathrm{h}$.

Si Silica

$T \quad$ Reaction temperature, $\mathrm{K}$

$W \quad$ Catalyst weight, $\mathrm{kg}$

WHSV Weight hourly space velocity, $\mathrm{h}^{-1}$

$W / F_{\text {Ao }} \quad$ Space time, $\mathrm{kg} . \mathrm{h} / \mathrm{kgmol}$ referred to isopropanol feed rate

$X_{\mathrm{A}} \quad$ Fractional conversion of isopropanol

Greek letters

$\Delta \quad$ change space time, $\mathrm{kg} \mathrm{h} / \mathrm{kgmol}$

Subscripts

A isopropanol

$A_{o} \quad$ feed isopropanol

a apparent

$f$ formation

$p \quad$ pressure

\section{Acknowledgements}

The author is grateful to Council of Scientific and Industrial Research (CSIR), New Delhi, India, for a Senior Research Fellowship during the tenure of this work. The author is also grateful to Dr C V Satyanarayana, Catalysis Division (NCL), Pune for the experimental facilities.

\section{References}

1. Ismailov R G, Aliev S M, Guseinov N I, Akhmed Zade Z A and Guseinov R I 1973 Khim. Tekhnol. Topl. Masel 186

2. Burress G T, Kaeding W W, Wu M M, Young L B 1980 US Pat. 4197413

3. Young L B 1983 US Pat. 4375573

4. Flockhart B D, Liew K Y and Pink R C $1981 J$. Catal. 72314

5. Upadhyayula S 2008 Can. J. Chem. Eng. 86207

6. Allen R H and Yats L D 1960 J. Amer. Chem. Soc. 82 4853

7. Olah G A, Kuhn S J and Flood S H 1961 J. Amer. Chem. Soc. 836541

8. Fraenkel D and Levy M 1989 J. Catal. 11810 
9. Parikh P A, Subrahmanyam N, Bhat Y S and Halgeri A B 1992 Appl. Catal. A90 1

10. Guo H, Han C and Wang X 1993 Shiyou Xuebao Shiyou Jiagong 941

11. Čejka J, Kapustin G A and Witchterlová B 1994 Appl. Catal. A108 187

12. Reddy K S N, Rao B S and Shiralkar V P 1995 Appl. Catal. A121 191

13. Witchterlová B, Čejka J and Zilkova N 1996 Microporous Mater. 6405

14. Lok B M, Messina C A, Patton R L, Gajek R T, Cannan T R and Flanigen E M 1984 US Pat. 4440871
15. Tapp N J, Milestone N B and Bibby D M 1988 Innovation in zeolite materials science, studies in surface science and catalysis (eds) P J Grobet et al (Amsterdam: Elsevier) vol.37, p. 393

16. Yue P L and Olaofe O 1984 Chem. Eng. Res. Des. 62 167

17. Parikh P A, Subrahmanyam N, Bhat Y S and Halgeri A B 1991 Chem. Eng. J. 5479

18. Bhat Y S, Halgeri A B and Prasada Rao T S R 1989 Ind. Eng. Chem. Res. 28890

19. Reid R C, Prausnitz J M and Sherwood T K 1977 The properties of gases and liquids (New York: McGraw Hill) p. 248 